\title{
Analysis and Comparison of Methods Evaluation Process Multifactor Simple Additive Weighting Method In Tilawatil Musabaqah Quran (MTQ) North Sumatra Province
}

\author{
Heriyanto $^{1}$, Olven Manahan ${ }^{2}$ \\ Informatics Engineering Program, STMIK Pelita Nusantara, Jl. St. Iskandar Muda No. 1 \\ Medan, Nort Sumatera, Indonesia, 20154 \\ E-mail: rian.penusa@my.gavilan.edu ${ }^{1}$,olven_manahan79@yahoo.com²
}

\begin{abstract}
The purpose of holding an MTQ is Essentially to broadcast the Koran while looking for the best to represent the MTQ at the next level. But the point is the greatness of newspapers. With that symbol, it IS ALSO DEMANDED that a person is not only good at reading and writing, but far more important is understanding the content of the newspaper itself. The goal is not only for of participants (who take part in the competition) but Also (and this is more important) for the listener. Decision Support System (DSS) is used as an alternative to application in the selection of MusabaqahTilawatil Quran (MTQ) in North SumatraProvince by using the multifactor Evaluation Process Method and the Simple Additive Weighting Method. In the MFEP and SAW methods decision making is done by giving Subjective and objective considerations Considered to factors that are important. Reviews These considerations are in the form of weighting the multifactor Involved and are Considered important. With this Analysis and Comparison, it is expected to be Able to find a more suitable method and Be Able to help as a jury assistant to take a decision on the contestants and can help solve the problems in the MusabaqahTilawatil Quran (MTQ) Selection in North Sumatra Province properly.
\end{abstract}

Keywords: Musabaqah Tilawatil Quran (MTQ), multifactor Evaluation Process, Simple Additive weighting

\section{Introduction}

Musabaqah Tilawatil Qur'an (MTQ) First held in 1940 since the founding of Jami'iyah Qurro wal Huffadz established by Nahdlatul Ulama, the largest Islamic organizations in Indonesia. [1] Since 1968, when the ministers of religion held by KH Muhammad Dahlan MTQ instituted nationwide. MTQ first held in Maksar during Ramadan in 1968. [1] MTQ until 2019 it had lasted a total of 27 times and MTQ 27 carried in Medan, North Sumatra. [1] Systematic calculation of the value of the participants in the implementation of Musabaqah Tilawatil Qur'an is the thing to the integrated and accountable results and decisions. To the authors conducted a study to compare two methods of multifactor Evaluation Process (MFEP) and Simple Additive weighting method (SAW) in order to determine which method is more suitable and efficient in implementing the MTQ assessment system.

\section{Research methodology}

\subsection{Multi method Factro Evaluation Process (MFEP)}

Multifactor Evaluation Process a decision-making model that uses a collective approach dariproses decision making [2] As for the calculation process steps using MFEP methods, namely:

a) Determining factors and weighting factors where the total weighting must be equal to one.

b) Load value for each factor affecting the decision of the data to be processed, the value entered in the decision-making process of the data to be processed, the value entered in the decision making process is an objective value.

c) Evaluation of weight calculation process is a process between the weight calculation of weight factor and factor evaluation by the sum of all evaluations hasilweight untukmemperoleh total evaluation results.

$\mathbf{W}=\mathbf{w 1}+\mathbf{w} 2+\mathrm{w} 3+\mathrm{w} 4+$ .Persamaan wn (1)

Information :

$\mathrm{W}=$ Total weight criteria

$\mathrm{w}=$ Weight of criteria 
We $=$ we .Persamaan

Information :

We $=$ Evaluation weights

$\mathrm{w}=$ Weight of criteria

$\mathrm{e}=$ Evaluation criteria

\section{Evaluation $=$ Weight Weight Factor $\mathbf{x}$ Factor Evaluation}

Multi-factor in decision making, decision makers subjectively and intuitively weigh the various factors that have an important influence on alternative them.For influence strategic decisions, it is recommended to use a quantitative approach as MFEP [2].

In the first MFEP - all around the criteria that an important factor in the consideration given weighting (weighting) as appropriate. The same step conducted on alternative - the alternative will be selected, who then dapatdievaluasi related to factors - factors such consideration. Jumlahdarimasing - masingbobotkriteria (w) harussamadengan 1 and mempunyairange nilaievaluasikriteria (e).

\subsection{Simple Additive weighting method (SAW)}

Simple Additive weighting method (SAW) is often also known summation method terbobot.Konsep term basis SAW method is to find a weighted summation of rating performance on each alternative on all attributes. SAW method requires a process of normalizing the decision matrix $(\mathrm{X})$ to a scale that can be compared with all the ratings of existing alternatives. This method is the method most famous and most widely used in dealing with situations of Multiple Attribute Decision Making (MADM) [3] .MADM itself is a method used to find alternatives alternativeoptimal of a number of specific criteria.

SAW method requires decision makers determining the weights for each attribute. The total score for alternatives is obtained by adding up all the multiplication of rating (which can be compared cross-attribute) and the weight of each attribute. Rating each attribute dimension must be free in the sense that has gone through the process of normalization previous matrix [3].

Step Completion Simple Additive Weighting (SAW) as follows [5]:

a) Determining criteria - criteria that will be used as reference in decision making.

b) The rating determines the suitability of each alternative on each criterion.

c) Make decisions based on criteria matrix (Ci), then normalized matrix based on equations that are tailored to the type attribute (attribute or attributes benefit costs) in order to obtain the normalized matrix R.

d) The final results obtained from the ranking process is the summation of the normalized $\mathrm{R}$ matrix multiplication with the weight vector in order to obtain the greatest value is selected as the best alternative (Ai) as a solution.

formula untukmelakukannormalisasitersebutadalah :

$$
r_{i j}=\frac{\frac{\mathrm{X}_{\mathrm{ij}}}{\operatorname{Max}_{\mathrm{i}} \mathrm{X}_{\mathrm{ij}}} \mathrm{Jika} \mathrm{j} \text { adalah Atribut keuntungan (benefit) }}{\frac{\operatorname{Min}_{\mathrm{i}} \mathrm{X}_{\mathrm{ij}}}{\mathrm{X}_{\mathrm{ij}}} \text { jika } \mathrm{j} \text { adalah atribut biaya cost }}
$$

Where :

rij $=$ Normalized performance rating

Maxij = Maximum value of each row and column

Minij = Minimum value of each row and column

$\mathrm{Xij}=$ rows and columns of a matrix

With rij is the normalized performance rating of alternatives on attribute $\mathrm{Ai} \mathrm{Cj} ; \mathrm{i}=1,2, \ldots \mathrm{m}$ and $\mathrm{j}=1,2, \ldots$, n. Preference value for each alternative (Vi) is given as:

$$
\mathrm{V}_{i}=w_{j=1} r_{i j}
$$

Where :

$\mathrm{Vi}=$ Nilaiakhir of alternative

wj $=$ The weights have been determined 
rij $=$ Normalization matrix

Vi larger value indicates that the alternative chosen.

2.3 Stage Research

In conducting this study the authors conducted several stages for collecting and managing data include:

a) Doing Literature

The author conducted a study related to the research literature to get more information and references

b) Observation and data collection

The author conducted a case study, the research by conducting observations and interviews to the practitioner or other authorized bodies of research to get the data needed in the research process.

c) Data management

The author obtained and selecting the data that is needed for use in research.

\section{Results and Discussion}

Analysis and comparison of two methods will result in a decision in the form of advice to the Council of Justice to take the winner Decision MTQ.

In Musabaqah Tilawatil Qur'an there are several criteria including:

Table 1.

\begin{tabular}{ccl}
\multicolumn{3}{c}{ criteria MTQ } \\
\hline No. & Criteria & \multicolumn{1}{c}{ Information } \\
\hline 1 & C1 & AdabFashohah \\
2 & C2 & tajwid \\
3 & C3 & Song \\
4 & C4 & Sound \\
\hline
\end{tabular}

Table 2

weights Preferences

\begin{tabular}{|c|c|c|c|}
\hline No. & Description & Score & Weight \\
\hline 1 & Very good & $96-100$ & 1 \\
\hline 2 & Well & $91-95$ & 0.75 \\
\hline 3 & Enough & $81-90$ & 0.5 \\
\hline 4 & Less & $\leq 80$ & 0.25 \\
\hline
\end{tabular}

Table 3

Table Weight Criteria

\begin{tabular}{clc}
\hline No. & \multicolumn{1}{c}{ Criteria } & Weight \\
\hline 1 & AdabFashohah & $25 \%$ \\
2 & tajwid & $35 \%$ \\
3 & Song & $20 \%$ \\
4 & Sound & $20 \%$ \\
\hline
\end{tabular}

Table 4

Results of Conversion Value Into weights Alternative Preferences

\begin{tabular}{llcccc} 
No. & \multicolumn{1}{c}{ Name } & C1 & C2 & C3 & C4 \\
1 & Mohammed Sidik & 1 & 0.75 & 0.25 & 0.5 \\
2 & EkoKuswoyo & 0.75 & 1 & 0.25 & 0.75 \\
3 & DianyarSaidatulHusna & 0.25 & 0.25 & 0.75 & 1 \\
4 & Muhamad Ari & 0.5 & 0.25 & 0.5 & 1 \\
5 & Risky Gani & 0.75 & 1 & 0.25 & 0.5 \\
6 & M. Agung Permana & 1 & 0.25 & 0.5 & 0.75 \\
7 & Sari Latifah Salma & 0.25 & 0.75 & 1 & 0.25 \\
\hline
\end{tabular}




\subsection{Calculation Method MFEP}

And the formula for calculating the Total Weight Evaluation is by adding up all the multiplication of Weight Factor with Factor Evaluation and can be described as follows:

Total Weight Evaluation $=\Sigma$ Weight Factor $x$ Factor Evaluation

Calculation of Total Weight Evaluation will be conducted on each participant MTQ registered and participants who had the highest Total Weight Evaluation will be the champion. Berikutmerupakanhasilperhitunganberdasarkan sample data contained in Tabel3.4 albeit from masingpesrta:

Table 5

Total Weight Evaluation Mohammed Sidik

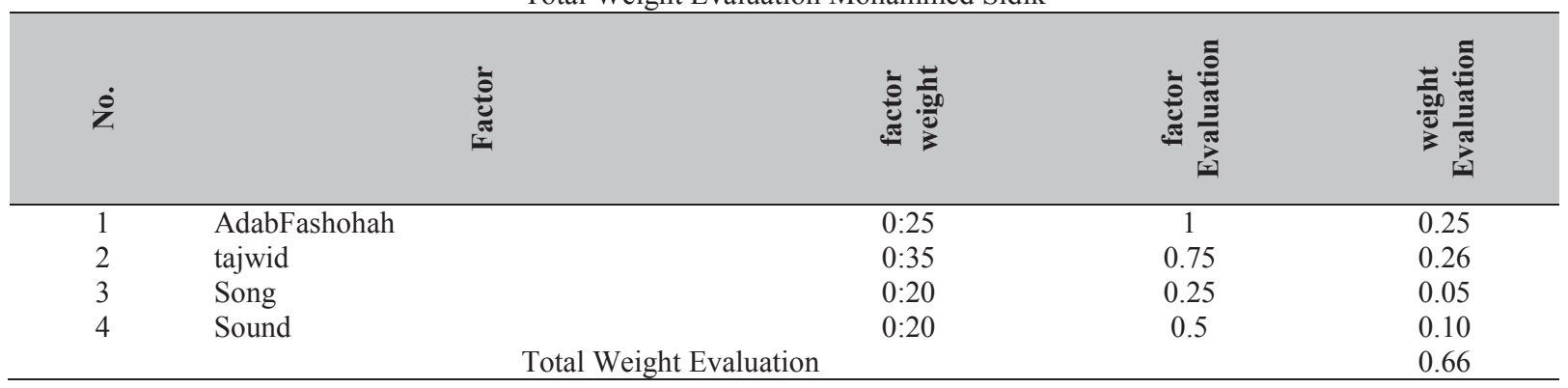

Table 6

Total Weight Evaluation EkoKuswoyo

\begin{tabular}{|c|c|c|c|c|}
\hline$\dot{0}$ & 节 & 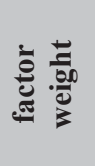 & 竞 & 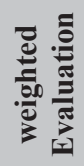 \\
\hline $\begin{array}{l}1 \\
2 \\
3 \\
4\end{array}$ & $\begin{array}{l}\text { AdabFashohah } \\
\text { tajwid } \\
\text { Song } \\
\text { Sound }\end{array}$ & $\begin{array}{c}0: 25 \\
0: 35 \\
0: 20 \\
0: 20 \\
\text { luation }\end{array}$ & $\begin{array}{c}0.75 \\
1 \\
0.25 \\
0.75\end{array}$ & $\begin{array}{l}0.19 \\
0.35 \\
0.05 \\
0.15 \\
0.74 \\
\end{array}$ \\
\hline
\end{tabular}

Table 7

Total Weight Evaluation Dianyar Saidatul Husna

\begin{tabular}{|c|c|c|c|c|}
\hline$\dot{z}$ & 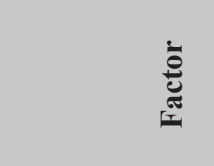 & 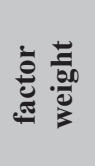 & 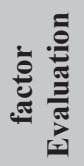 & 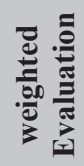 \\
\hline $\begin{array}{l}1 \\
2 \\
3 \\
4\end{array}$ & $\begin{array}{l}\text { AdabFashohah } \\
\text { tajwid } \\
\text { Song } \\
\text { Sound }\end{array}$ & $\begin{array}{c}0: 25 \\
0: 35 \\
0: 20 \\
0: 20 \\
\text { luation }\end{array}$ & $\begin{array}{c}0.25 \\
0.25 \\
0.75 \\
1\end{array}$ & $\begin{array}{l}0.06 \\
0.09 \\
0.15 \\
0.20 \\
0.50\end{array}$ \\
\hline
\end{tabular}

Table 8

Total Weight Evaluation Mohammed Ari

\begin{tabular}{|c|c|c|c|c|}
\hline$\dot{z}$ & 离 & 冚 & 竞 & 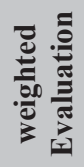 \\
\hline 1 & AdabFashohah & $0: 25$ & 0.5 & 0.13 \\
\hline 2 & tajwid & $0: 35$ & 0.25 & 0.09 \\
\hline 3 & Song & $0: 20$ & 0.5 & 0.10 \\
\hline 4 & Sound & $0: 20$ & 1 & 0.20 \\
\hline \multicolumn{4}{|c|}{ Total Weight Evaluation } & 0.51 \\
\hline
\end{tabular}


Tabel.9

Total Weight Evaluation Risky Gani

\begin{tabular}{|c|c|c|c|c|}
\hline$\dot{z}$ & 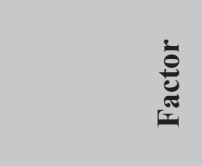 & 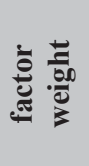 & 竞 & 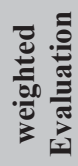 \\
\hline $\begin{array}{l}1 \\
2 \\
3 \\
4\end{array}$ & $\begin{array}{l}\text { AdabFashohah } \\
\text { tajwid } \\
\text { Song } \\
\text { Sound }\end{array}$ & $\begin{array}{c}0: 25 \\
0: 35 \\
0: 20 \\
0: 20 \\
\text { luation }\end{array}$ & $\begin{array}{c}0.75 \\
1 \\
0.25 \\
0.5\end{array}$ & $\begin{array}{l}0.19 \\
0.35 \\
0.05 \\
0.10 \\
0.69 \\
\end{array}$ \\
\hline
\end{tabular}

Table 10

Total Weight Evaluation M. Agung Permana

\begin{tabular}{|c|c|c|c|c|}
\hline$\stackrel{0}{z}$ & 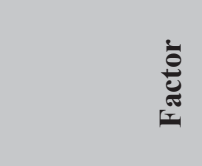 & 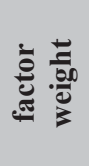 & 竞 & 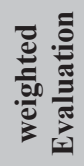 \\
\hline $\begin{array}{l}1 \\
2 \\
3 \\
4\end{array}$ & $\begin{array}{l}\text { AdabFashohah } \\
\text { tajwid } \\
\text { Song } \\
\text { Sound }\end{array}$ & $\begin{array}{c}0: 25 \\
0: 35 \\
0: 20 \\
0: 20 \\
\text { luation } \\
\end{array}$ & $\begin{array}{c}1 \\
0.25 \\
0.5 \\
0.75\end{array}$ & $\begin{array}{l}0.25 \\
0.09 \\
0.10 \\
0.15 \\
0.59 \\
\end{array}$ \\
\hline
\end{tabular}

Table 11

Weight Evaluation Sari Latifah Salma

\begin{tabular}{|c|c|c|c|c|}
\hline$\dot{8}$ & 总 & 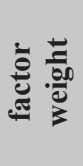 & 竎 & 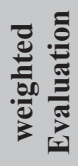 \\
\hline 1 & AdabFashohah & $0: 25$ & 0.25 & 0.06 \\
\hline 2 & tajwid & $0: 35$ & 0.75 & 0.26 \\
\hline 3 & Song & $0: 20$ & 1 & 0.20 \\
\hline 4 & Sound & $0: 20$ & 0.25 & 0.05 \\
\hline \multicolumn{4}{|c|}{ Total Weight Evaluation } & 0.58 \\
\hline
\end{tabular}

The final results of the calculation method for the competitor MTQ MFEP which has the largest value that the winner of the race, the decision can be seen in the table below:

Table 12

Decision Methods MFEP

\begin{tabular}{llll}
\hline No. & \multicolumn{1}{c}{ Name } & TWE & Information \\
\hline 1 & Mohammed Sidik & 0662 & ranking 3 \\
2 & EkoKuswoyo & 0745 & ranking 2 \\
3 & DianyarSaidatulHusna & 0504 & - \\
4 & Muhamad Ari & 0515 & - \\
5 & Risky Gani & 0693 & ranking 2 \\
6 & M. Agung Permana & 0595 & - \\
7 & Sari Latifah Salma & 0581 & - \\
\hline
\end{tabular}




\subsection{Calculation Method SAW}

Normalization Adab Fashohah (C1)

$r_{11}=\frac{\mathrm{X}_{11}}{\max \mathrm{X}_{11}, \mathrm{X}_{21}, \mathrm{X}_{31}, \mathrm{X}_{41}}=\frac{1}{1 ; 0,75 ; 0,25 ; 0,5}=1$
$r_{12}=\frac{\mathrm{X}_{12}}{\max \mathrm{X}_{12}, \mathrm{X}_{22}, \mathrm{X}_{32}, \mathrm{X}_{42}}=\frac{0,75}{0,75 ; 1 ; 0,25 ; 0,75}=0,75$
$r_{13}=\frac{\mathrm{X}_{13}}{\max \mathrm{X}_{13}, \mathrm{X}_{23}, \mathrm{X}_{33}, \mathrm{X}_{43}}=\frac{0,25}{0,25 ; 0,25 ; 0,75 ; 1}=0,25$
$r_{14}=\frac{\mathrm{X}_{14}}{\max \mathrm{X}_{14}, \mathrm{X}_{24}, \mathrm{X}_{34}, \mathrm{X}_{44}}=\frac{0,5}{0,5 ; 0,25 ; 0,5 ; 1}=0,5$
$r_{15}=\frac{\mathrm{X}_{15}}{\max \mathrm{X}_{15}, \mathrm{X}_{25}, \mathrm{X}_{35}, \mathrm{X}_{45}}=\frac{0,75}{0,75 ; 1 ; 0,25 ; 0,5}=0,75$
$r_{16}=\frac{\mathrm{X}_{16}}{\max \mathrm{X}_{16}, \mathrm{X}_{26}, \mathrm{X}_{36}, \mathrm{X}_{46}}=\frac{1}{1 ; 0,25 ; 0,5 ; 0,75}=1$
$r_{17}=\frac{\mathrm{X}_{17}}{\max \mathrm{X}_{17}, \mathrm{X}_{27}, \mathrm{X}_{37}, \mathrm{X}_{47}} \quad=\frac{0,25}{0,25 ; 0,75 ; 1 ; 0,25}=0,25$

Normalization Tajweed (C2)

$r_{21}=\frac{\mathrm{X}_{21}}{\max \mathrm{X}_{11}, \mathrm{X}_{21}, \mathrm{X}_{31}, \mathrm{X}_{41}}$

$=\frac{0,75}{1 ; 0,75 ; 0,25 ; 0,5}=0,75$

$r_{22}=\frac{\mathrm{X}_{22}}{\max \mathrm{X}_{12}, \mathrm{X}_{22}, \mathrm{X}_{32}, \mathrm{X}_{42}}$

$=\frac{1}{0,75 ; 1 ; 0,25 ; 0,75}=1$

$r_{23}=\frac{\mathrm{X}_{23}}{\max \mathrm{X}_{13}, \mathrm{X}_{23}, \mathrm{X}_{33}, \mathrm{X}_{43}}$

$=\frac{0,25}{0,25 ; 0,25 ; 0,75 ; 1}=0,25$

$r_{24}=\frac{\mathrm{X}_{24}}{\max \mathrm{X}_{14}, \mathrm{X}_{24}, \mathrm{X}_{34}, \mathrm{X}_{44}}$

$=\frac{0,5}{0,5 ; 0,25 ; 0,5 ; 1}=0,50$

$r_{25}=\frac{\mathrm{X}_{25}}{\max \mathrm{X}_{15}, \mathrm{X}_{25}, \mathrm{X}_{35}, \mathrm{X}_{45}}$

$r_{26}=\frac{\mathrm{X}_{26}}{\max \mathrm{X}_{16}, \mathrm{X}_{26}, \mathrm{X}_{36}, \mathrm{X}_{46}}$

$r_{27}=\frac{\mathrm{X}_{27}}{\max \mathrm{X}_{17}, \mathrm{X}_{27}, \mathrm{X}_{37}, \mathrm{X}_{47}}$

$=\frac{1}{0,75 ; 1 ; 0,25 ; 0,5}=1$

$=\frac{0,25}{1 ; 0,25 ; 0,5 ; 0,75}=0,25$

$=\frac{0,75}{0,25 ; 0,75 ; 1 ; 0,25}=0,75$

Voice Normalization (C3)

$$
\begin{aligned}
& r_{31}=\frac{\mathrm{X}_{31}}{\max \mathrm{X}_{11}, \mathrm{X}_{21}, \mathrm{X}_{31}, \mathrm{X}_{41}} \\
& =\frac{0,25}{1 ; 0,75 ; 0,25 ; 0,5}=0,25 \\
& r_{32}=\frac{\mathrm{X}_{32}}{\max \mathrm{X}_{12}, \mathrm{X}_{22}, \mathrm{X}_{32}, \mathrm{X}_{42}} \\
& =\frac{0,25}{0,75 ; 1 ; 0,25 ; 0,75}=0,25 \\
& r_{33}=\frac{\mathrm{X}_{33}}{\max \mathrm{X}_{13}, \mathrm{X}_{23}, \mathrm{X}_{33}, \mathrm{X}_{43}} \\
& =\frac{0,75}{0,25 ; 0,25 ; 0,75 ; 1}=0.75 \\
& r_{34}=\frac{\mathrm{X}_{34}}{\max \mathrm{X}_{14}, \mathrm{X}_{24}, \mathrm{X}_{34}, \mathrm{X}_{44}}=\frac{0,5}{0,5 ; 0,25 ; 0,5 ; 1}=0.5 \\
& r_{35}=\frac{\mathrm{X}_{35}}{\max \mathrm{X}_{15}, \mathrm{X}_{25}, \mathrm{X}_{35}, \mathrm{X}_{45}}=\frac{0,25}{0,75 ; 1 ; 0,25 ; 0,5}=0,25 \\
& r_{36}=\frac{\mathrm{X}_{36}}{\max \mathrm{X}_{16}, \mathrm{X}_{26}, \mathrm{X}_{36}, \mathrm{X}_{46}}=\frac{0,5}{1 ; 0,25 ; 0,5 ; 0,75}=0,50 \\
& r_{37}=\frac{\mathrm{X}_{37}}{\max \mathrm{X}_{17}, \mathrm{X}_{27}, \mathrm{X}_{37}, \mathrm{X}_{47}} \quad=\frac{1}{0,25 ; 0,75 ; 1 ; 0,25}=1
\end{aligned}
$$

\section{Normalization Songs (C4)}


$r_{41}=\frac{\mathrm{X}_{41}}{\max \mathrm{X}_{11}, \mathrm{X}_{21}, \mathrm{X}_{31}, \mathrm{X}_{41}} \quad=\frac{0,5}{1 ; 0,75 ; 0,25 ; 0,5}=0,50$
$r_{42}=\frac{\mathrm{X}_{42}}{\max \mathrm{X}_{12}, \mathrm{X}_{22}, \mathrm{X}_{32}, \mathrm{X}_{42}} \quad=\frac{0,75}{0,75 ; 1 ; 0,25 ; 0,75}=0,75$
$r_{43}=\frac{\mathrm{X}_{43}}{\max \mathrm{X}_{13}, \mathrm{X}_{23}, \mathrm{X}_{33}, \mathrm{X}_{43}} \quad=\frac{1}{0,25 ; 0,25 ; 0,75 ; 1}=1$
$r_{44}=\frac{\mathrm{X}_{44}}{\max \mathrm{X}_{14}, \mathrm{X}_{24}, \mathrm{X}_{34}, \mathrm{X}_{44}} \quad=\frac{1}{0,5 ; 0,25 ; 0,5 ; 1}=1$
$r_{45}=\frac{\mathrm{X}_{45}}{\max \mathrm{X}_{15}, \mathrm{X}_{25}, \mathrm{X}_{35}, \mathrm{X}_{45}} \quad=\frac{0,5}{0,75 ; 1 ; 0,25 ; 0,5}=0,50$
$r_{46}=\frac{\mathrm{X}_{46}}{\max \mathrm{X}_{16}, \mathrm{X}_{26}, \mathrm{X}_{36}, \mathrm{X}_{46}} \quad=\frac{0,75}{1 ; 0,25 ; 0,5 ; 0,75}=0,75$
$r_{47}=\frac{\mathrm{X}_{47}}{\max \mathrm{X}_{17}, \mathrm{X}_{27}, \mathrm{X}_{37}, \mathrm{X}_{47}} \quad=\frac{0,25}{0,25 ; 0,75 ; 1 ; 0,25}=0,25$

Table 13

normalized matrix

\begin{tabular}{llcccc} 
No. & \multicolumn{1}{c}{ Name } & C1 & C2 & C3 & C4 \\
\hline 1 & Mohammed Sidik & 1 & 0.75 & $0: 25$ & 0.5 \\
2 & EkoKuswoyo & 0.75 & 1 & $0: 25$ & 0.75 \\
3 & DianyarSaidatulHusna & $0: 25$ & $0: 25$ & 0.75 & 1 \\
4 & Muhamad Ari & 0.5 & $0: 25$ & 0.5 & 1 \\
5 & Risky Gani & 0.75 & 1 & $0: 25$ & 0.5 \\
6 & M. Agung Permana & 1 & $0: 25$ & 0.5 & 0.75 \\
7 & Sari Latifah Salma & $0: 25$ & 0.75 & 1 & $0: 25$ \\
\hline
\end{tabular}

After giving the value of each weight further criteria to calculate the value perengkingan preference for each alternative $(\mathrm{Vi})$ with the following formula:

Where:

$$
\mathrm{V}_{i}=w_{j=1} w_{j} r_{i j}
$$

$\mathrm{Vi}=$ The final value of the alternative

$\mathrm{wj}=$ Weights telahditentukan

rij $=$ Normalisasimatriks

Vi larger value indicates that the competitor who will pass the selection race in kecamatan Perbaungan MTQ to the formula as below:

$$
\begin{aligned}
& \text { V1 } \quad=(0.25 * 1)+(0.35 * 0.75)+(0.20 * 0.25)+(0.20 * 0.5)=.663 \\
& \text { V2 }=(0.25 * 0.75)+(0.35 * 1)+(0.20 * 0.25)+(0.20 * 0.75)=0.738 \\
& \text { V3 }=(0.25 * 0.25)+(0.35 * 0.25)+(0.20 * 0.75)+(0.20 * 1)=0.550 \\
& \text { V4 }=(0.25 * 0.5)+(0.35 * 0.25)+(0.20 * 0.5)+(0.20 * 1)=0.513 \\
& \text { V5 }=(0.25 * 0.75)+(0.35 * 1)+(0.20 * 0.25)+(0.20 * 0.5)=0.688 \\
& \text { V6 }=(0.25 * 1)+(0.35 * 0.25)+(0.20 * 0.5)+(0.20 * 0.75)=0.588 \\
& \text { V7 }=(0.25 * 0.25)+(0.35 * 0.75)+(0.20 * 1)+(0.20 * 0.25)=0.575
\end{aligned}
$$

Thus a calculated above makadapat drawn the conclusion that the value of the largest value becomes the winner of MTQ, using SAW algorithm as in the table below.

Table 14

Decision SAW Method

\begin{tabular}{llcl}
\hline No. & \multicolumn{1}{c}{ Name } & Vi & Information \\
\hline 1 & Mohammed Sidik & 0663 & ranking 3 \\
2 & EkoKuswoyo & $\mathbf{0 7 3 8}$ & ranking 1 \\
3 & DianyarSaidatulHusna & 0500 & - \\
4 & Muhamad Ari & 0513 & - \\
5 & Risky Gani & $\mathbf{0 6 8 8}$ & ranking 2 \\
6 & M. Agung Permana & 0588 & - \\
7 & Sari Latifah Salma & 0575 & - \\
\hline
\end{tabular}




\section{Conclusion}

From the above data it can be concluded that the method of SAW and MFEP have an accurate calculation. But for MFEP method has the steps - steps which is much shorter than the SAW method, it proves that the SAW method can be made to become the alternative valuation methods because the level of accuracy is much higher than the MFEP method. This is caused by the process of doing the calculation method of SAW in the normalization process in which this is not done on MFEP method.

\section{Reference}

[1]. https://id.wikipedia.org/wiki/MTQ diakses tanggal 28 Januari 2019

[2]. Kusrini, M.Kom. (2007). Konsep Dan Aplikasi Sistem Pendukung Keputusan. Yogyakarta: ANDI.

[3] Kusumadewi,S dkk. (2006).Fuzzy Multiatribute Decision Making. Jogjakarta:Graha Ilmu.

[4]. Rosa.A.S, (2014). Pemodelan sistem Rekayasa perangkat lunak. Jakarta: PT. Elex Media Komputindo

[5]. Dicky Nofriansyah, M.Kom (2014). Konsep Data Mining Vs Sistem Pendukung Keputusan. Yokyakarta: Cv Budi Utama) 